\title{
A numerical approach for calculation of characteristics of edge waves in three dimensional plates
}

\author{
Wenbo Duan,"; Ray Kirby ${ }^{\mathrm{b}}$ \\ ${ }^{a}$ School of Engineering and Computer Science, University of Hertfordshire, UK. AL10 9AB; \\ w.duan@herts.ac.uk \\ ${ }^{\mathrm{b}}$ Centre for Audio, Acoustics and Vibration, University of Technology Sydney, Sydney, NSW, 2007 \\ Australia
}

\begin{abstract}
Surface waves have been extensively studied in earthquake seismology. Surface waves are trapped near an infinitely large surface. The displacements decay exponentially with depth. These waves are also named Rayleigh and Love waves. Surface waves are also used for non-destructive testing of surface defects. Similar waves exist in finite-width three dimensional plates. In this case, displacements are no longer constant in the direction perpendicular to the wave propagation plane. Wave energy could still be trapped near the edge of the three dimensional plate, and hence the term edge waves. These waves are thus different to the two dimensional Rayleigh and Love waves. This article presents a numerical model to study dispersion properties of edge waves in plates. A two dimensional semianalytical finite element method is developed, and the problem is closed by a perfectlymatched-layer adjacent to the edge. The numerical model is validated by comparing with available analytical and numerical solutions in the literature. On this basis, higher order edge waves and mode shapes are presented for a three dimensional plate. The characteristics of the presented edge wave modes could be used in non-destructive testing applications.
\end{abstract}

1.Introduction. 
Theoretical studies on surface waves can be traced back to late nineteenth century, for instance, Lord Rayleigh investigated Rayleigh waves travelling along the free surface of a semi-infinite half-space [1]. Love predicted the existence of another type of surface waves, where particle displacement is perpendicular to the wave propagation plane[2]. Love waves could also be named as shear-horizontal surface waves. These waves are well studied in the literature, and three dimensional governing equations are normally simplified as two dimensional equations, because of the assumption of constant displacements in one direction. However, surface waves could also form near the edge of a finite width plate, and displacements are no longer constant in the width direction due to reflections from edges. These waves are thus named edge waves. An analytical solution of edge waves involves displacements in three dimensions, and these analytical solutions are generally limited to specific edge modes. Full dispersion analysis of edge waves hasn't been presented in the literature. In this article, a two-dimensional semi-analytical finite element method is developed to study dispersion properties of edge waves in plates. The numerical solutions are validated by comparing with available analytical and numerical solutions for surface and edge waves in the literature. On this basis, full dispersion curves of edge waves in a typical plate are presented, which include symmetric, flexural and higher order modes.

Analytical solutions have been developed for calculation of a fundamental flexural edge wave mode in semi-infinite plates [3-5]. These early studies are based on the classical Kirchhoff thin plate theory, where the flexural wave motion is uncoupled to displacements in other two dimensions. Lagasse and Oliner [6] verified the existence of the flexural mode using finite element calculations and experimental measurements. It was found that the thin plate theory is only valid in the very low frequency range. However, the development of surface wave theories requires the plate height to be infinitely large compared to the plate width, to avoid reflection of waves coming from the bottom of the plate. This is not possible in the 
conventional finite element model proposed by Lagasse and Oliner, and thus this numerical model is not suitable for calculation of full edge wave modes. Various plate theories have been proposed for the development of flexural edge wave modes in orthotropic and submerged conditions etc [7-16]. Krushynska [17] presented an exact analytical solution for dispersion analysis of flexural edge waves in isotropic plates. The superposition method is used to find the solution of the displacement potential equations, based on the three dimensional equations of linear elasticity.

However, flexural edge waves are only part of the solution for the three dimensional wave equations. It is well known that for Lamb waves in isotropic plates, displacements could be either symmetric (extensional) or anti-symmetric (flexural). By analogy with Lamb waves, edge waves could also be extensional or flexural. Kaplunov et al [18] studied symmetric edge wave propagation in isotropic plates subject to mixed face boundary conditions. These mixed face boundary conditions are not natural boundary conditions, however, they facilitate the incorporation of trigonometric functions into displacements. Zernov and Kaplunov [19] later investigated the more general problem of symmetric edge wave propagation in plates with either stress free or fixed face boundary conditions. The symmetric edge waves are expressed as an infinite series of Rayleigh-Lamb and shear modes, which could be solved numerically. Edge resonance (or localised vibration) is another related but different topic. Edge resonance could occur when the symmetric Lamb mode S0 incident upon the free edge of a semiinfinite strip [20]. This topic is not further discussed here.

Edge phenomena also appear for guided waves travelling along welds or bends in plates. Postnova and Craster [21] proposed an asymptotic long-wave theory to study weld-guided waves in plates. Fan and Lowe [22] numerically and experimentally investigated edge waves guided by a weld joint in a plate. The semi-analytical finite element (SAFE) method is used to calculate the eigen solutions, and the absorbing region method is used to close the 
problem. The same approach was also used to study guided wave propagation along stiffeners bonded to plates [23]. Ramdhas et al [24] and Yu et al [25] showed that feature-guided waves appear for thin plates with transverse bends. The semi-analytical finite element (SAFE) method thus appears to be one of the most widely used methods for dispersion analysis of guided waves in plates and pipes etc [26-30]. These waveguides generally have an arbitrary but closed cross-sectional area. For open waveguides, i.e., infinitely large, immersed or buried waveguides etc, the problem can be tackled by combining the SAFE method with the perfectly matched layer (PML) method [31-34]. The PML method is used to stretch coordinates and in the same time damp wave radiation in the infinitely large surrounding medium.

Despite these analytical and numerical studies, full dispersion curves for edge waves in a three dimensional plate haven't been presented in the literature. In this article, a general weighted residual based SAFE-PML method is proposed to study edge wave propagation in plates. The same method could also be used to study wave propagation along welds, bends etc. The feature area is modelled using the SAFE method, and the area surrounding this feature is modelled using the PML method. Stress free boundary conditions have been assumed for all the structures studied in this article. The numerical model is validated by comparing current numerical solutions with Rayleigh, Love, and exactly flexural edge wave solutions in the literature. On this basis, full dispersion curves for edge waves on a three dimensional plate are presented.

2. Development of finite element equations 
The configuration of a three dimensional plate is shown in Fig. 1. The plate is assumed to be infinitely long in the wave propagation direction, i.e., $-\infty<z<\infty$. The plate has a width $b$ $(-b / 2 \leq x \leq b / 2)$, and semi-infinite in the thickness direction $(y \leq 0)$. Edge waves decay exponentially in the thickness direction. The SAFE method requires only the cross-sectional area of the plate to be meshed. To avoid meshing an infinitely large area in the thickness direction, the PML method is used to close the problem. It is assumed that the PML layer starts at $y=-h$, and finishes at $y=-2 h$, so that the thickness of the PML layer is $h$.

In the SAFE layer, the governing equations could be written in the Cartesian coordinates as:

$$
\rho_{p} \frac{\partial^{2} u_{j}^{*}}{\partial t^{2}}=\frac{\partial \sigma_{j x}^{*}}{\partial x}+\frac{\partial \sigma_{j y}^{*}}{\partial y}+\frac{\partial \sigma_{j z}^{*}}{\partial z}, \quad j=x, y \text { or } z
$$

Here, $\rho_{p}$ is density, $t$ is time, $u_{j}^{*}$ is displacement in the $j$ direction $(j=x, y$ or $z)$, and $\sigma_{j l}^{*}(j, l=x, y$ or $z)$ is the elastic normal or shear stress. A time dependence of $e^{\mathrm{i} \omega t}$ is assumed throughout this paper, where $\omega$ is the radian frequency and $i=\sqrt{-1}$. The plate could include an arbitrary number of layers, however, each layer is assumed to be homogeneous and isotropic. 


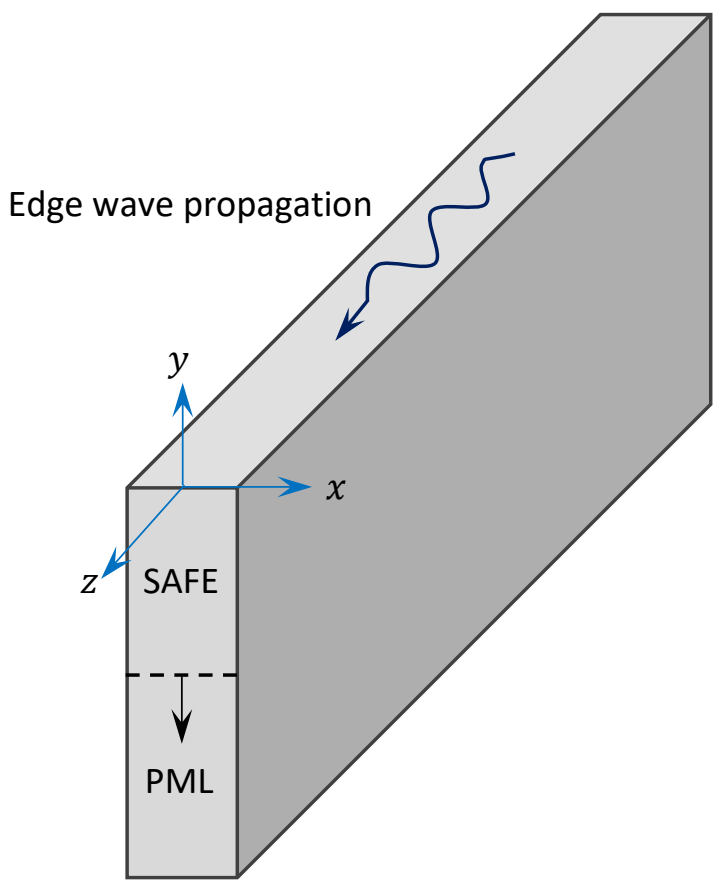

Fig. 1. Edge wave propagation along the top surface of a plate.

As shown in Fig. 1, the wave propagates in the $z$ direction, and thus the displacements could be written in the form:

$$
u_{j}^{*}(x, y, z)=u_{j}(x, y) e^{i(\omega t-k \gamma z)}, \quad j=x, y \text { or } z
$$

Here, $\gamma$ is a dimensionless wavenumber, $k=\omega / c_{\mathrm{T}}$, and $c_{\mathrm{T}}$ is the shear bulk wave velocity. The Galerkin's principle based weighted residual method is used in this paper. Substitution of Eq. (2) into Eq. (1), the weak forms of Eq. (1) can be developed through integration by parts and making use of the usual relationships between stress and strain : 


$$
\begin{aligned}
& \int_{\Omega_{p}}\left\{\left(\lambda_{p} \frac{\partial w_{x}}{\partial x} \frac{\partial u_{x}}{\partial x}+2 \mu_{p} \frac{\partial w_{x}}{\partial x} \frac{\partial u_{x}}{\partial x}+\mu_{p} \frac{\partial w_{x}}{\partial y} \frac{\partial u_{x}}{\partial y}+(k \gamma)^{2} \mu_{p} w_{x} u_{x}\right.\right. \\
& \left.-\rho_{p} \omega^{2} w_{x} u_{x}\right)+\left(\lambda_{p} \frac{\partial w_{x}}{\partial x} \frac{\partial u_{y}}{\partial y}+\mu_{p} \frac{\partial w_{x}}{\partial y} \frac{\partial u_{y}}{\partial x}\right) \\
& \left.+\left(-i k \gamma \lambda_{p} \frac{\partial w_{x}}{\partial x} u_{z}+i k \gamma \mu_{p} w_{x} \frac{\partial u_{z}}{\partial x}\right)\right\} d \Omega_{p}=\int_{\Gamma_{p}} w_{x} h_{x} d \Gamma_{p} \\
& \int_{\Omega_{p}}\left\{\left(\lambda_{p} \frac{\partial w_{y}}{\partial y} \frac{\partial u_{x}}{\partial x}+\mu_{p} \frac{\partial w_{y}}{\partial x} \frac{\partial u_{x}}{\partial y}\right)\right. \\
& +\left(\lambda_{p} \frac{\partial w_{y}}{\partial y} \frac{\partial u_{y}}{\partial y}+\mu_{p} \frac{\partial w_{y}}{\partial x} \frac{\partial u_{y}}{\partial x}+2 \mu_{p} \frac{\partial w_{y}}{\partial y} \frac{\partial u_{y}}{\partial y}+(k \gamma)^{2} \mu_{p} w_{y} u_{y}\right. \\
& \left.\left.-\rho_{p} \omega^{2} w_{y} u_{y}\right)+\left(-i k \gamma \lambda_{p} \frac{\partial w_{y}}{\partial y} u_{z}+i k \gamma \mu_{p} w_{y} \frac{\partial u_{z}}{\partial y}\right)\right\} d \Omega_{p} \\
& =\int_{\Gamma_{p}} w_{y} h_{y} d \Gamma_{p} \\
& \int_{\Omega_{p}}\left\{\left(i k \gamma \lambda_{p} w_{z} \frac{\partial u_{x}}{\partial x}-i k \gamma \mu_{p} \frac{\partial w_{z}}{\partial x} u_{x}\right)+\left(i k \gamma \lambda_{p} w_{z} \frac{\partial u_{y}}{\partial y}-i k \gamma \mu_{p} \frac{\partial w_{z}}{\partial y} u_{y}\right)\right. \\
& +\left(\mu_{p} \frac{\partial w_{z}}{\partial x} \frac{\partial u_{z}}{\partial x}+\mu_{p} \frac{\partial w_{z}}{\partial y} \frac{\partial u_{z}}{\partial y}+(k \gamma)^{2} \lambda_{p} w_{z} u_{z}+(k \gamma)^{2} 2 \mu_{p} w_{z} u_{z}\right. \\
& \left.\left.-\rho_{p} \omega^{2} w_{z} u_{z}\right)\right\} d \Omega_{p}=\int_{\Gamma_{p}} w_{z} h_{z} d \Gamma_{p}
\end{aligned}
$$

Here, $\lambda_{p}$ and $\mu_{p}$ are the Lamé coefficients in the SAFE layer. $w_{j}(j=x, y$ or $z)$ is an arbitrary weighting function to be determined later. $\Omega_{p}$ is the cross-sectional area of the SAFE layer, and $\Gamma_{p}$ is the boundary of $\Omega_{p} \cdot h_{j}=\sigma_{j x} n_{x}+\sigma_{j y} n_{y}(j=x, y$ or $z)$ is the external traction, and $n_{j}$ is the unit normal on the boundary of $\Omega_{p}$.

In the PML layer, the governing equations could be written as:

$$
\rho_{m} \frac{\partial^{2} u_{j}^{*}}{\partial t^{2}}=\frac{1}{\xi_{x}} \frac{\partial \sigma_{j x}^{*}}{\partial x}+\frac{1}{\xi_{y}} \frac{\partial \sigma_{j y}^{*}}{\partial y}+\frac{\partial \sigma_{j z}^{*}}{\partial z}, j=x, y \text { or } z
$$


where $\xi_{x}$ and $\xi_{y}$ are nowhere-zero, continuous, complex-valued coordinate stretching functions. Eq. (6) is written in a general form, which could incorporate coordinate stretching in both $x$ and $y$ directions. For the conventional problem of edge wave propagation in the plate shown in Fig. 1, the $x$ direction coordinate remains unchanged, so that $\xi_{x}=1$.

Eq. (6) is multiplied by $\xi_{x} \xi_{y}$, and the weak forms of the governing equations can be derived using integration by parts as:

$$
\begin{aligned}
& \int_{\Omega_{m}}\left\{\left(\lambda_{m} \frac{\xi_{y}}{\xi_{x}} \frac{\partial w_{x}}{\partial x} \frac{\partial u_{x}}{\partial x}+2 \mu_{m} \frac{\xi_{y}}{\xi_{x}} \frac{\partial w_{x}}{\partial x} \frac{\partial u_{x}}{\partial x}+\mu_{m} \frac{\xi_{x}}{\xi_{y}} \frac{\partial w_{x}}{\partial y} \frac{\partial u_{x}}{\partial y}\right.\right. \\
& \left.+(k \gamma)^{2} \mu_{m} \xi_{x} \xi_{y} w_{x} u_{x}-\rho_{m} \omega^{2} \xi_{x} \xi_{y} w_{x} u_{x}\right) \\
& +\left(\lambda_{m} \frac{\partial w_{x}}{\partial x} \frac{\partial u_{y}}{\partial y}+\mu_{m} \frac{\partial w_{x}}{\partial y} \frac{\partial u_{y}}{\partial x}\right) \\
& \left.+\left(-i k \gamma \lambda_{m} \xi_{y} \frac{\partial w_{x}}{\partial x} u_{z}+i k \gamma \mu_{m} \xi_{y} w_{x} \frac{\partial u_{z}}{\partial x}\right)\right\} d \Omega_{m} \\
& =\int_{\Gamma_{m}} w_{x} \sum_{j=x, y}\left(\frac{\xi_{x} \xi_{y}}{\xi_{j}} \sigma_{i j} n_{j}\right) d \Gamma_{m} \\
& \int_{\Omega_{m}}\left\{\left(\lambda_{m} \frac{\partial w_{y}}{\partial y} \frac{\partial u_{x}}{\partial x}+\mu_{m} \frac{\partial w_{y}}{\partial x} \frac{\partial u_{x}}{\partial y}\right)\right. \\
& +\left(\lambda_{m} \frac{\xi_{x}}{\xi_{y}} \frac{\partial w_{y}}{\partial y} \frac{\partial u_{y}}{\partial y}+\mu_{m} \frac{\xi_{y}}{\xi_{x}} \frac{\partial w_{y}}{\partial x} \frac{\partial u_{y}}{\partial x}+2 \mu_{m} \frac{\xi_{x}}{\xi_{y}} \frac{\partial w_{y}}{\partial y} \frac{\partial u_{y}}{\partial y}\right. \\
& \left.+(k \gamma)^{2} \mu_{m} \xi_{x} \xi_{y} w_{y} u_{y}-\rho_{m} \omega^{2} \xi_{x} \xi_{y} w_{y} u_{y}\right) \\
& \left.+\left(-i k \gamma \lambda_{m} \xi_{x} \frac{\partial w_{y}}{\partial y} u_{z}+i k \gamma \mu_{m} \xi_{x} w_{y} \frac{\partial u_{z}}{\partial y}\right)\right\} d \Omega_{m} \\
& =\int_{\Gamma_{m}} w_{y} \sum_{j=x, y}\left(\frac{\xi_{x} \xi_{y}}{\xi_{j}} \sigma_{i j} n_{j}\right) d \Gamma_{m}
\end{aligned}
$$




$$
\begin{aligned}
\int_{\Omega_{m}}\left\{\left(\xi_{y} i k \gamma \lambda_{m}\right.\right. & \left.w_{z} \frac{\partial u_{x}}{\partial x}-\xi_{y} i k \gamma \mu_{m} \frac{\partial w_{z}}{\partial x} u_{x}\right) \\
& +\left(\xi_{x} i k \gamma \lambda_{m} w_{z} \frac{\partial u_{y}}{\partial y}-\xi_{x} i k \gamma \mu_{m} \frac{\partial w_{z}}{\partial y} u_{y}\right) \\
& +\left(\frac{\xi_{y}}{\xi_{x}} \mu_{m} \frac{\partial w_{z}}{\partial x} \frac{\partial u_{z}}{\partial x}+\frac{\xi_{x}}{\xi_{y}} \mu_{m} \frac{\partial w_{z}}{\partial y} \frac{\partial u_{z}}{\partial y}+\xi_{x} \xi_{y}(k \gamma)^{2} \lambda_{m} w_{z} u_{z}\right. \\
& \left.\left.+\xi_{x} \xi_{y}(k \gamma)^{2} 2 \mu_{m} w_{z} u_{z}-\xi_{x} \xi_{y} \rho_{m} \omega^{2} w_{z} u_{z}\right)\right\} d \Omega_{m} \\
& =\int_{\Gamma_{m}} w_{z} \sum_{j=x, y}\left(\frac{\xi_{x} \xi_{y}}{\xi_{j}} \sigma_{i j} n_{j}\right) d \Gamma_{m}
\end{aligned}
$$

Here, $\lambda_{m}$ and $\mu_{m}$ are the Lamé coefficients in the PML layer. It is possible that the plate includes a number of layers with different material properties in each layer. In this case, Lamé coefficients and density shall be changed accordingly in each layer, however, the governing equations remain the same forms. The displacements are now discretised using the conventional finite element method. Furthermore, isoparametric finite elements are used, so that weighting functions equal shape functions. Stress free boundary conditions are used on the exterior surface of the plate, and displacements and stresses are continuous across the interface between adjacent layers. Eqs. (3-5) and Eqs. (7-9) can be combined to form a systematic equation in the matrix form as:

$$
\begin{gathered}
{\left[\begin{array}{cccccc}
Z_{1 x} & Z_{1 y} & 0 & 0 & 0 & Z_{1 z} \\
Z_{2 x} & Z_{2 y} & 0 & 0 & 0 & Z_{2 z} \\
0 & 0 & Z_{3 z} & Z_{3 x} & Z_{3 y} & 0 \\
0 & 0 & 0 & I & 0 & 0 \\
0 & 0 & 0 & 0 & I & 0 \\
0 & 0 & 0 & 0 & 0 & I
\end{array}\right]\left\{\begin{array}{c}
u_{x} \\
u_{y} \\
u_{z} \\
i k \gamma u_{x} \\
i k \gamma u_{y} \\
i k \gamma u_{z}
\end{array}\right\}=} \\
i k \gamma\left[\begin{array}{cccccc}
0 & 0 & 0 & F_{x} & 0 & 0 \\
0 & 0 & 0 & 0 & F_{x} & 0 \\
0 & 0 & 0 & 0 & 0 & -F_{z} \\
I & 0 & 0 & 0 & 0 & 0 \\
0 & I & 0 & 0 & 0 & 0 \\
0 & 0 & I & 0 & 0 & 0
\end{array}\right]\left\{\begin{array}{c}
u_{x} \\
u_{y} \\
u_{z} \\
i k \gamma u_{x} \\
i k \gamma u_{y} \\
i k \gamma u_{z}
\end{array}\right\}
\end{gathered}
$$


Here, $I$ is an identity matrix of order $p_{q}$, and $p_{q}$ is the total number of nodes in the SAFEPML region. The rest of the matrices in Eq. (10) are given in the appendix. Equation (10) is a sparse eigenequation. Solution of the equation delivers complex wavenumbers and mode shapes as a function of frequency.

Eigenmodes include both edge (or surface) and radiation waves. Radiation waves are standing (resonance) waves in the PML region and these waves have little theoretical or practical significance. To separate the radiation waves from edge waves, the method proposed by Nguyen et al. [31] is used here. This is based on the kinetic energy ratio between the SAFE layer and the PML layer [32].

\section{Validation of the numerical model}

The finite element model is first validated here by comparing current numerical solutions with Rayleigh, Love and edge wave solutions in the literature. Rayleigh and Love waves have constant displacements in the width direction (i.e., $-\infty<x<\infty$ ). This can be done by setting the $x$ direction derivatives to be zero in Eq. (10). Note that in an analytical approach the $x$ displacement is often assumed to be zero for Rayleigh wave solutions. This would simplify the development of equations. In analogy, both $y$ and $z$ displacements are assumed to be zero for Love wave solutions. However, this simplification means that Rayleigh and Love wave solutions couldn't be obtained at the same time. In this paper, such simplification is not carried out in order to calculate both Rayleigh and Love wave solutions using Eq. (10).

Rayleigh waves have been extensively studied in seismology, where a continent could be considered as a stratified half-space consisting of a stack of homogeneous and isotropic 
elastic layers. Foti presented Rayleigh wave dispersion curves for the case of a layer on top of a homogenous half-space [35], using the method of reflection and transmission coefficients [36]. This case is repeated here for comparison. The top layer is $10 \mathrm{~m}$ thick with a compressional wave speed of $600 \mathrm{~m} / \mathrm{s}$, a shear wave speed of $300 \mathrm{~m} / \mathrm{s}$, and a density of 1800 $\mathrm{kg} / \mathrm{m}^{3}$. The bottom layer is a half-space with a compressional wave speed of $800 \mathrm{~m} / \mathrm{s}$, a shear wave speed of $400 \mathrm{~m} / \mathrm{s}$, and a density of $1800 \mathrm{~kg} / \mathrm{m}^{3}$. In this paper, the top layer is meshed using the SAFE method, and the bottom layer is meshed using the PML method. The thickness of the PML layer is set to be $10 \mathrm{~m}$. The coordinate in the PML layer is stretched in the vertical y direction only. The exponential stretching function proposed by Duan et al [32] is used here:

$$
\xi_{y}=e^{\alpha \bar{y}}-\mathrm{i}\left[e^{\beta \bar{y}}-1\right],
$$

where $\bar{y}=(|y|-h) / h, \alpha$ and $\beta$ are real valued constants. Values of $\alpha=3$ and $\beta=4$ are used in this paper. Convergence studies in terms of PML length, stretching coefficients etc have been performed, and general conclusions could be seen in [32]. For calculation of Rayleigh and Love waves, three-noded isoparametric line elements are used, and the model is implemented in MATLAB ${ }^{\circledR}$. The programme is executed using a laptop with four $2.6 \mathrm{GHz}$ Intel Core $^{\mathrm{TM}}$ CPU processors and 16 GB of RAM. 40 elements are used in the SAFE and PML region respectively. This delivers a fine element density with at least 16 nodes per wavelength up to $150 \mathrm{~Hz}$. The computation time is less than 2 s per frequency, including the calculation of energy velocity, separating surface waves from radiation waves, and sorting of modes.

Surface wave dispersion curves are calculated using Eq. (10) and are shown as grey and red solid lines in Fig. 2. Grey lines indicate Rayleigh waves and red lines indicate Love waves. 
Excellent agreement can be observed between current numerical solution and Rayleigh wave solution of Foti [35]. For this case, all the surface waves are trapped waves with a phase velocity less than the bulk shear velocity of the bottom layer. In addition to surface waves, leaky Lamb waves also exist. These waves have a phase velocity larger than the bulk shear wave velocity of the bottom layer. Leaky wave have an oscillation displacement pattern in the top layer, and energy leaks into the surrounding medium (the bottom half-space). These waves are not surface waves and are not reported here. A detailed discussion of leaky and trapped wave modes can be seen in $[31,32]$.

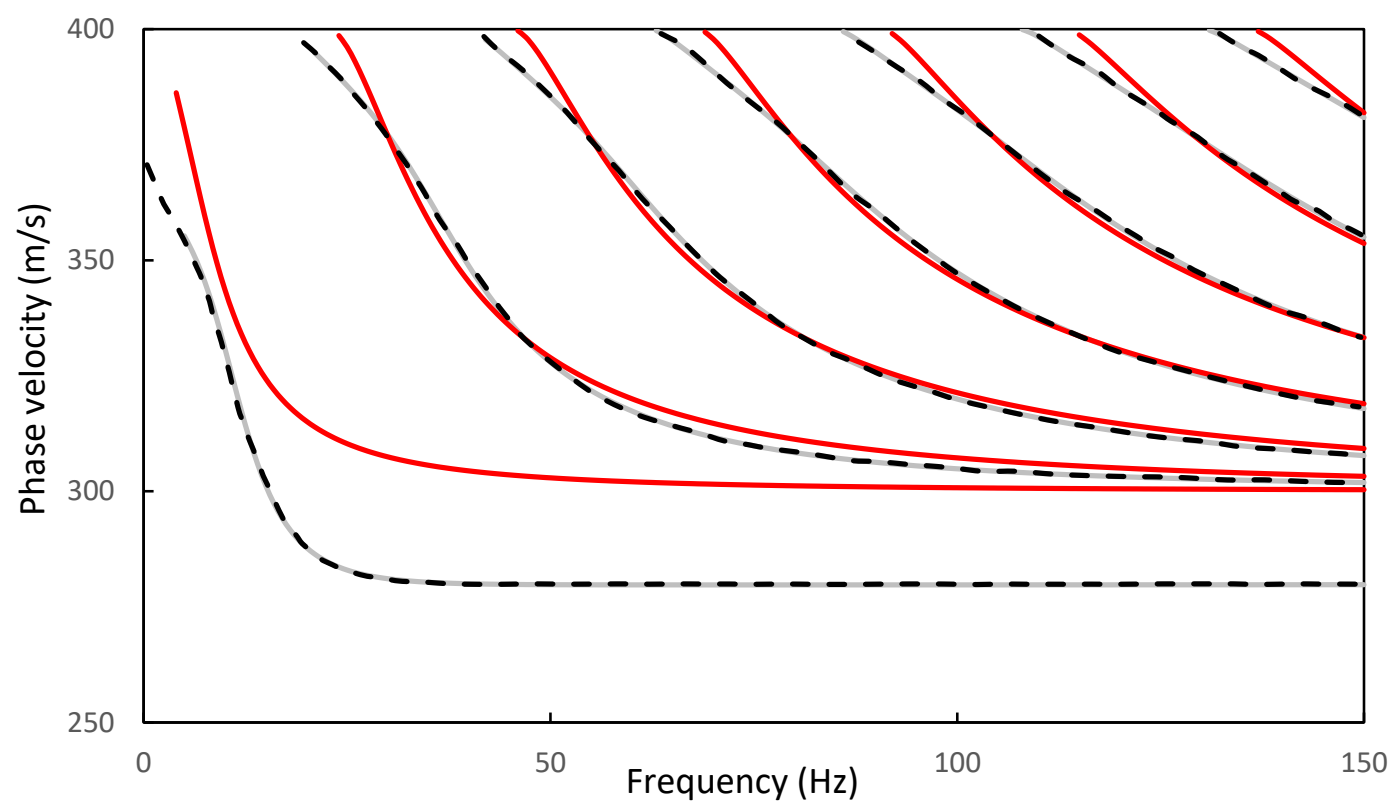

Fig. 2 Phase velocity dispersion curves. Solid lines: current numerical solution; dashed lines: Rayleigh wave solution of Foti (overlaid with the solid line) [35]. 
The current numerical model could be used to calculate Rayleigh, Love and leaky wave modes, which is generally not possible in an analytical approach. To further examine the accuracy of the numerical model, a second example is considered here. This is also a twolayer structure, and dispersion properties are presented in Fig. 3. Love wave solution of Eslick et al. [38] is also presented in Fig. 3 for comparison. Love waves have particle motion transverse to the direction of wave propagation, and it is thus possible to develop a simple dispersion relation for a two-layer system [39]. This relation is independent with compressional bulk wave velocities because of the shear horizontal nature of Love waves. However, for completeness of surface waves, compressional bulk wave velocities have been included in the numerical model. The top layer is $4 \mathrm{~m}$ thick with a compressional wave speed of $300 \mathrm{~m} / \mathrm{s}$, a shear wave speed of $150 \mathrm{~m} / \mathrm{s}$, and a density of $1600 \mathrm{~kg} / \mathrm{m}^{3}$. The bottom layer is a half-space with a compressional wave speed of $1000 \mathrm{~m} / \mathrm{s}$, a shear wave speed of $500 \mathrm{~m} / \mathrm{s}$, and a density of $1800 \mathrm{~kg} / \mathrm{m}^{3}$. The PML thickness if $4 \mathrm{~m}$. Twenty three-noded line elements are used in the SAFE and PML region respectively. Excellent agreement can be observed between the current numerical solution and Love wave solution of Eslick et al. Rayleigh wave solution has been presented as red lines in Fig. 3 for reference. These two independent examples (i.e., Fig. 2 and 3) show that the current numerical model is able to accurately calculate Rayleigh and Love wave solutions at the same time. 


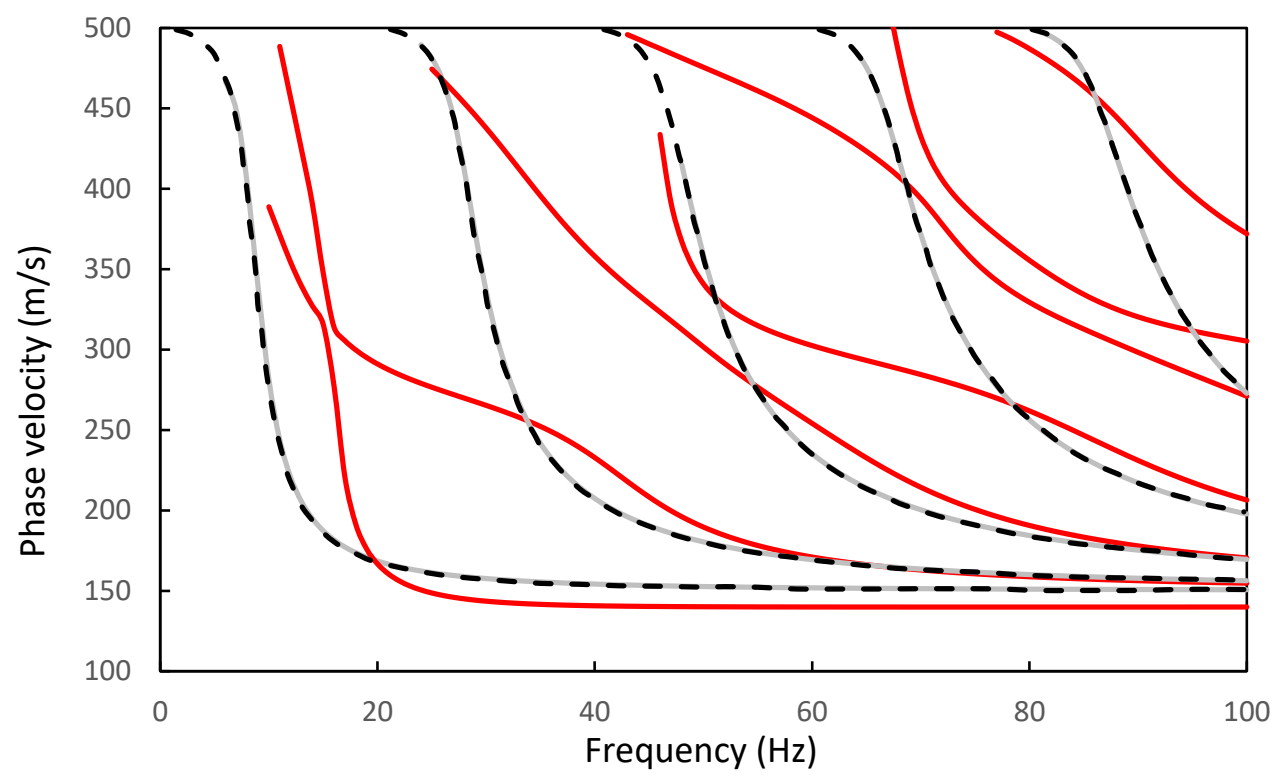

Fig. 3 Phase velocity dispersion curves. Solid lines: current numerical solution; dashed lines:

Love wave solution of Eslick et al (overlaid with the solid line) [38].

Rayleigh and Love waves have constant displacements in the width direction. These waves are easier to solve than edge waves in a three dimensional plate. Currently, analytical and numerical solutions for edge waves in a three dimensional plate are limited to individual symmetric or flexural wave modes. Fig. 4 shows the comparison between the current numerical solution and the analytical solution of Krushynska [17] for the fundamental flexural edge wave propagation in a plate. It has been assumed that the plate is isotropic and homogeneous. In the numerical model, the width of the plate is set to be $0.1 \mathrm{~m}$, i.e, $b=0.1$. The Young's modulus is $73 \mathrm{Gpa}$, the Poisson's ratio 0.39 , and the density $2800 \mathrm{~kg} / \mathrm{m}^{3}$. Traction free boundary conditions have been applied at all the surfaces. The phase velocity of the flexural edge wave has been normalised against Rayleigh wave velocity $c_{R}$ in an infinite half-space [17]. Non-dimensional wavenumber $\gamma$ of Rayleigh wave must satisfy the following secular equation [40]:

$$
4 \gamma^{2} \sqrt{\psi \gamma^{2}-1} \sqrt{\gamma^{2}-1}-\sqrt{\psi}\left(2 \gamma^{2}-1\right)^{2}=0
$$


Here, $\psi=\left(\lambda_{p}+2 \mu_{p}\right) / \mu_{p}$. Eq. (12) is independent with frequency. Upon numerical solution of Eq. (12), Rayleigh wave velocity can be obtained as $c_{R}=c_{\mathrm{T}} / \gamma$.

Fig. 4 shows excellent agreement between the current numerical solution and the analytical flexural edge wave solution of Krushynska [17]. In the numerical model, the height of the SAFE and PML layer is set to be $0.4 \mathrm{~m}$ respectively. Eight-noded quadratic rectangular elements are used, and the element size is $20 \mathrm{~mm}$. This ensures at least 20 nodes per wavelength at a frequency of $15 \mathrm{kHz}$. The total number of degrees of freedom is 2073 . The computation time is around 34 s per frequency, including separation of edge waves from radiation waves and sorting the modes. Clearly the current numerical model is able to calculate the edge wave accurately and efficiently. In analogy to extensional and flexural Lamb waves commonly found in a finite thickness plate, symmetric and higher order edge modes also exist in the semi-infinite plate, which are presented in the next section. 


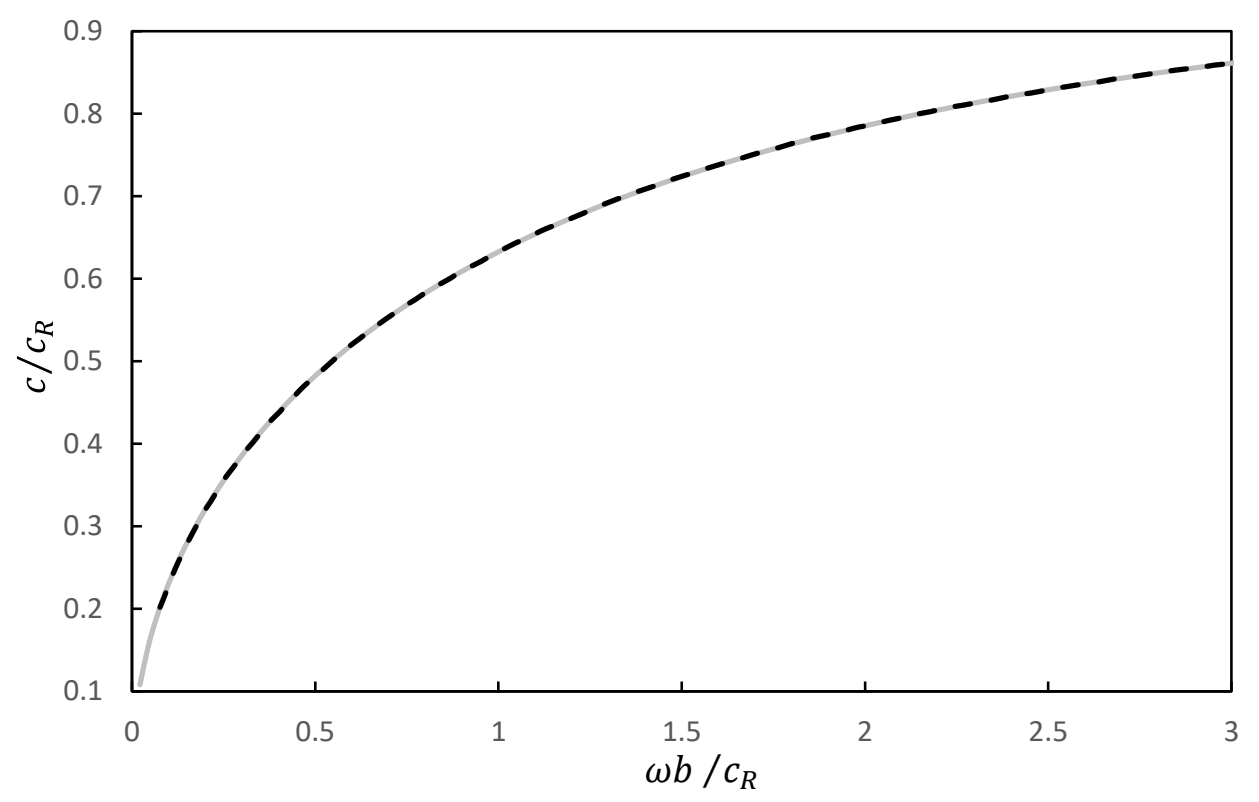

Fig. 4 Non-dimensional phase velocity of the fundamental flexural edge wave. Solid line: current numerical solution; dashed line: analytical solution of Krushynska (overlaid with the solid line) [17].

4 Edge waves in a three dimensional plate

Individual symmetric and flexural edge waves have been studied in the literature. However, to the best of the author's knowledge, complete dispersion analysis for edge waves in a three dimensional plate hasn't been conducted either analytically or numerically. The current numerical model is suitable to calculate dispersion curves for all edge waves in the plate, subjected to traction free boundary conditions. These dispersion curves are thus presented in this section. The three dimensional plate studied here is the same as the plate studied in the previous section, i.e., the width of the plate is $0.1 \mathrm{~m}$, the Young's modulus $73 \mathrm{Gpa}$, the Poisson's ratio 0.39 , and the density $2800 \mathrm{~kg} / \mathrm{m}^{3}$.

The flexural edge wave has been shown in Fig. 4. In this section, to study fundamental and higher order edge wave modes, the numerical model is implemented in the relative high frequency range from $10 \mathrm{kHz}$ to $120 \mathrm{kHz}$. The SAFE, PML thicknesses as well as 
corresponding finite element meshes have been modified in this section to balance the computation accuracy and efficiency of the model. Consequently, the height of the SAFE and PML layer is set to be $0.05 \mathrm{~m}$ respectively. Eight-noded quadratic rectangular elements are used, and the element size is $5 \mathrm{~mm}$. This ensures at least 10 nodes per wavelength at a frequency of $120 \mathrm{kHz}$. The total number of degrees of freedom is 3843 . The computation time is around $49 \mathrm{~s}$ per frequency. The edge wave modes are selected based on the kinetic energy ratio and attenuation ratio. Any mode with a kinetic energy ratio larger than 0.8 is considered as a radiation mode and discarded. The modes with an attenuation ratio larger than $30 \mathrm{~dB} / \mathrm{m}$ are also discarded.

Fig.5 presents phase velocity and attenuation curves for fundamental and higher order edge waves in the plate. The bulk shear wave velocity $c_{T}$ is also included in the figure for comparison. Edge modes are labelled as $\mathrm{E}_{\mathrm{Am}}$ or $\mathrm{E}_{\mathrm{Sm}}$ in this paper. The subscript $\mathrm{A}$ indicates a flexural wave mode, and subscript $S$ indicates a symmetric wave model. The subscript $m$ indicates mode sequence in each group. Fig. 5 (b) shows that fundamental edge waves are trapped wave modes with zero attenuation. However, higher order edge waves have energy leaking into the half-space in the $y$ direction. The attenuation ratio of the higher order symmetric mode $\mathrm{E}_{\mathrm{S} 1}$ is significantly lower than that of the higher order flexural mode $\mathrm{E}_{\mathrm{A} 1}$. It is also interesting to see that although phase velocities of higher order edge modes are lower than the bulk shear wave velocity of the plate in the high frequency range, these higher order modes are not trapped wave modes because displacements in different directions have different vertical wavenumbers. This means that some displacements are trapped while the others could be leaky. The characteristics of these edge wave modes can be seen in corresponding mode shapes. 

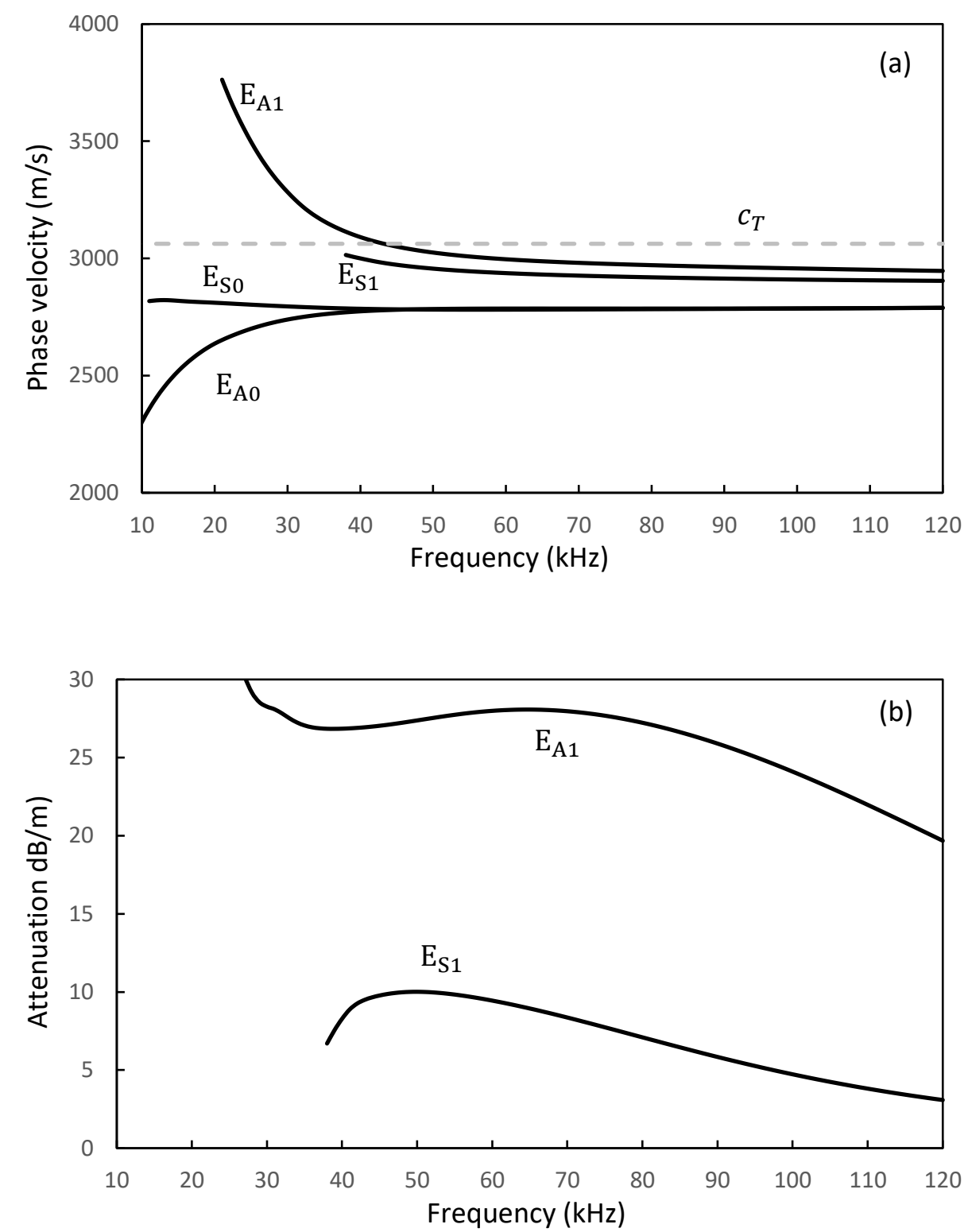

Fig. 5 Dispersion curves for edge waves in a three dimensional plate. (a) phase velocity; (b) attenuation.

Figs. 6-9 show the mode shapes of these four edge modes at $60 \mathrm{kHz}$. The height of the SAFE layer has been increased to $0.1 \mathrm{~m}$, and displacements in the PML layer are not shown because of artificial stretching and damping. The fundamental flexural mode $\mathrm{E}_{\mathrm{A} 0}$ has a displacement that is strongest in the $x$ direction (see Fig. 6). $x$ displacement of $\mathrm{E}_{\mathrm{A} 0}$ is anti-symmetric (i.e., 
flexural). All the three displacements are decaying in the vertical $y$ direction. At this frequency, the wavelength of the bulk shear wave is $51 \mathrm{~mm}$, less than the width of the plate. The edge wave has an apparent displacement variation in the width direction, and it can be seen that displacements are strongest near the corners of the plate on the top.

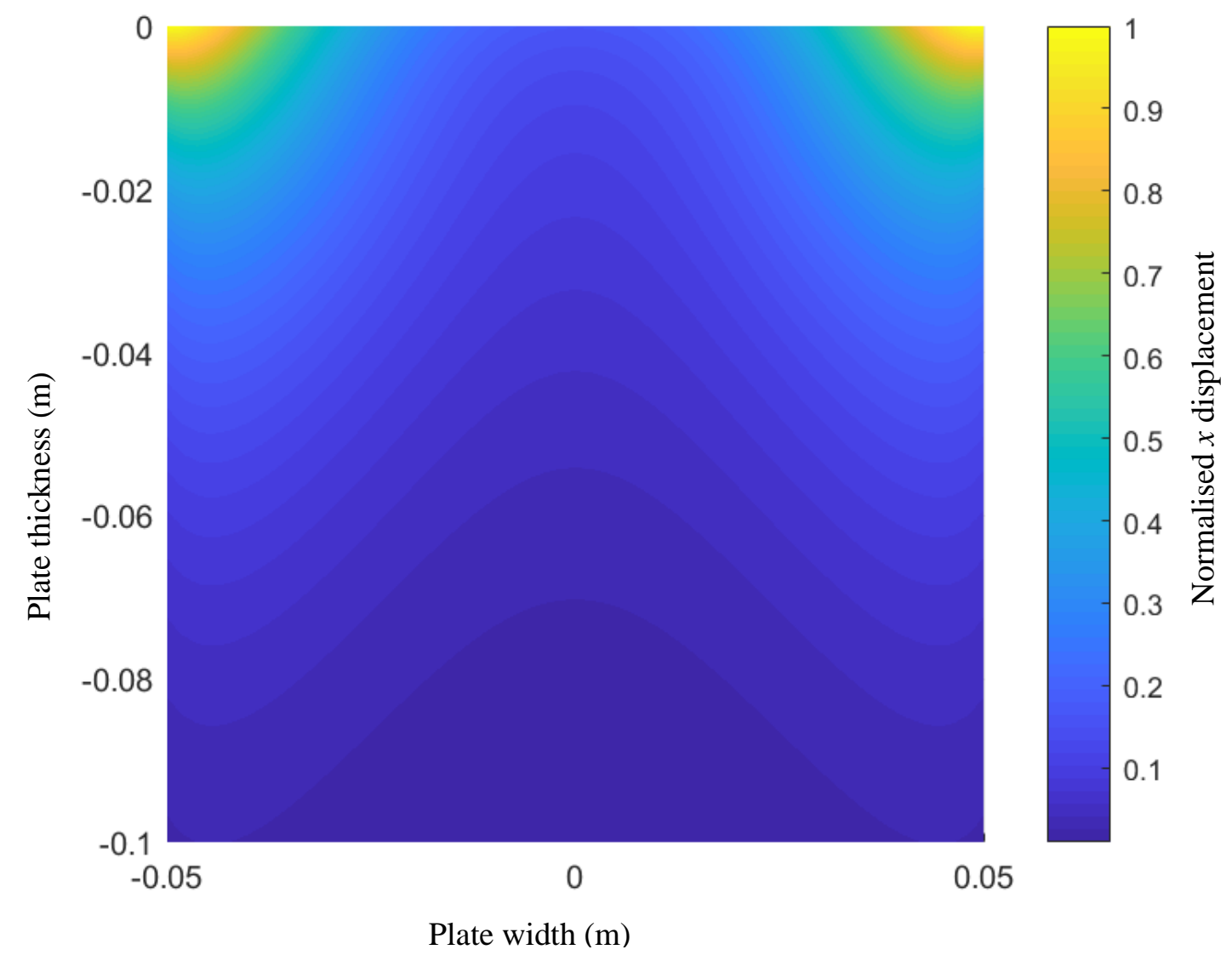




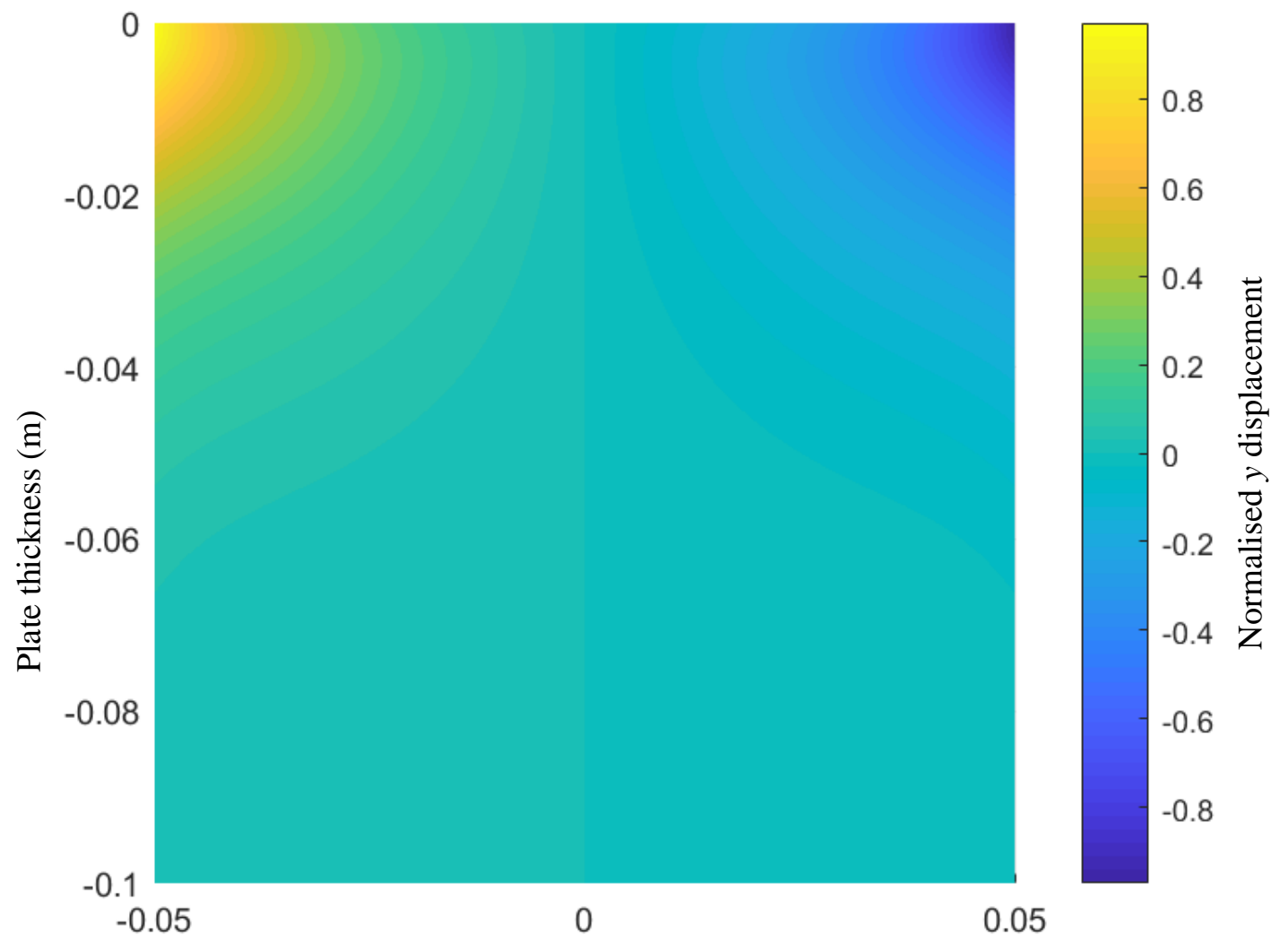

Plate width (m) 


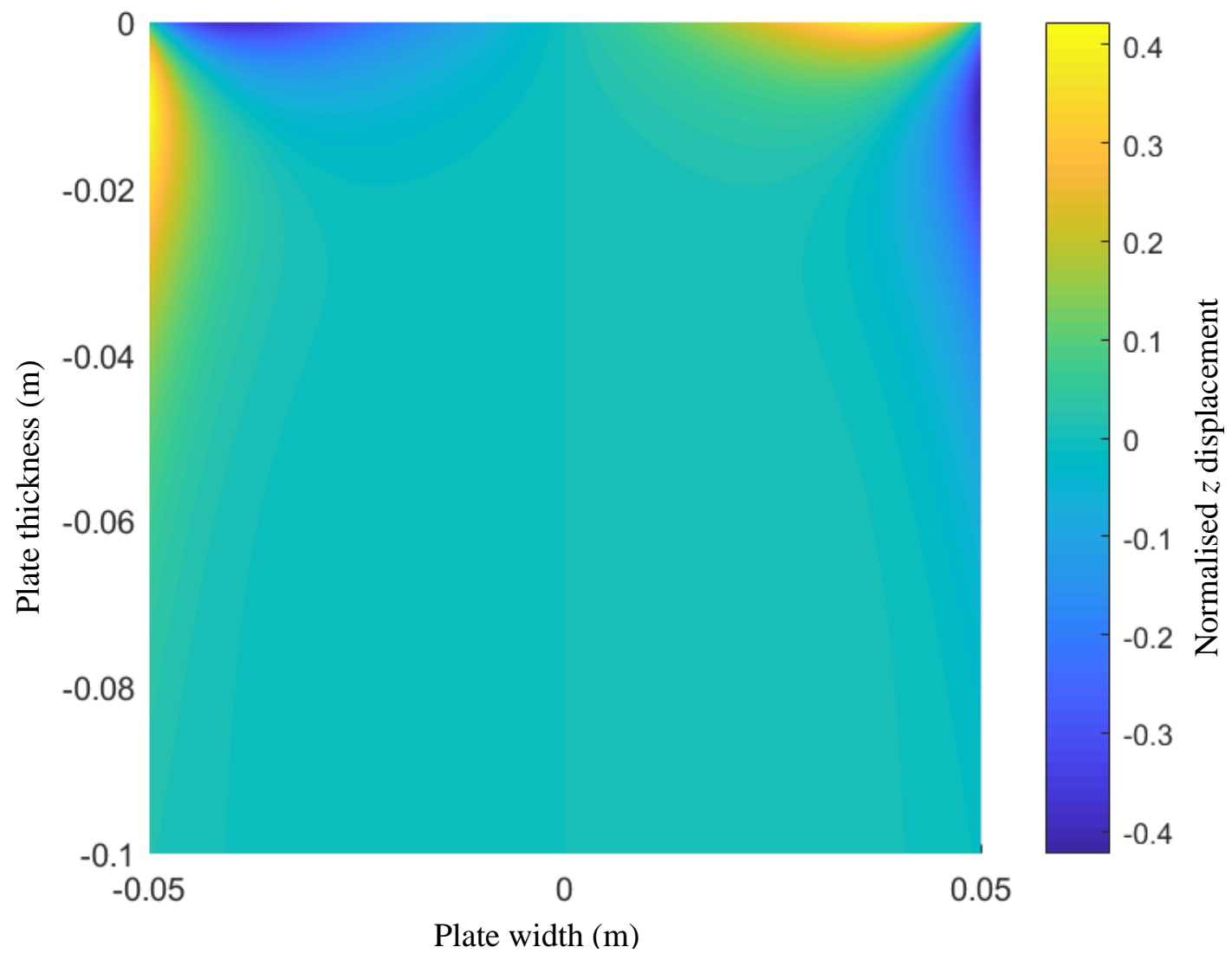

Fig. 6 Mode shape of the fundamental flexural edge wave $\mathrm{E}_{\mathrm{A} 0}$ at $60 \mathrm{kHz}$. (a) $x$ displacement;

(b) $y$ displacement; (c) $z$ displacement.

The fundamental symmetric edge mode $\mathrm{E}_{\mathrm{S} 0}$ has a displacement that is strongest in the $y$ direction (see Fig. 7). In contrast to $\mathrm{E}_{\mathrm{A} 0}, x$ displacement of $\mathrm{E}_{\mathrm{S} 0}$ is symmetric. Displacements are also strongest near the corners of the plate. Figs 6 and 7 show that $\mathrm{E}_{\mathrm{S} 0}$ and $\mathrm{E}_{\mathrm{A} 0}$ are a pair of wave modes with similar mode shapes and different symmetric patterns. These mode shape patterns are similar to conventional symmetric and anti-symmetric Lamb wave patterns in a plate, except that energy are focused near the top of the plate. 


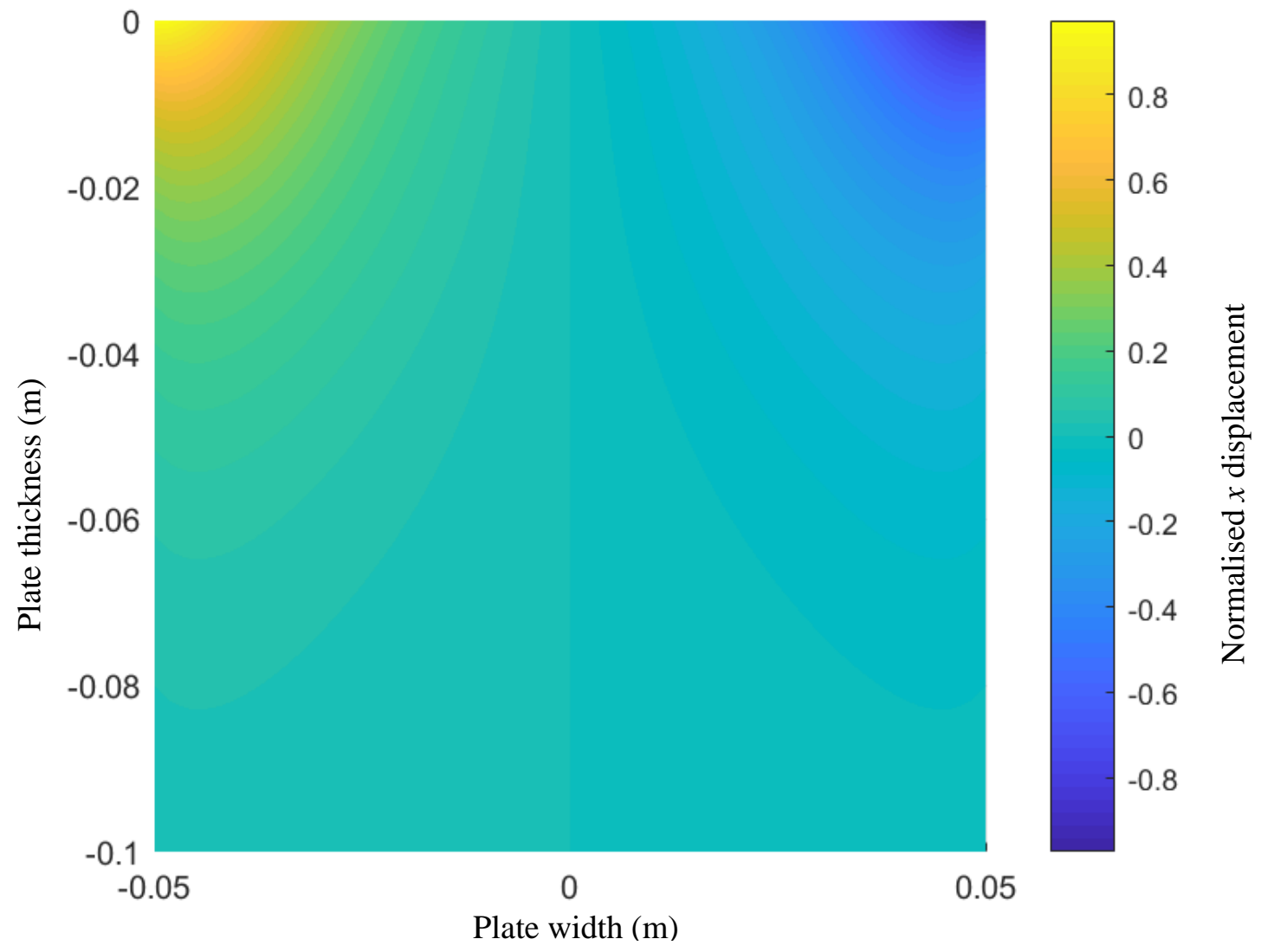




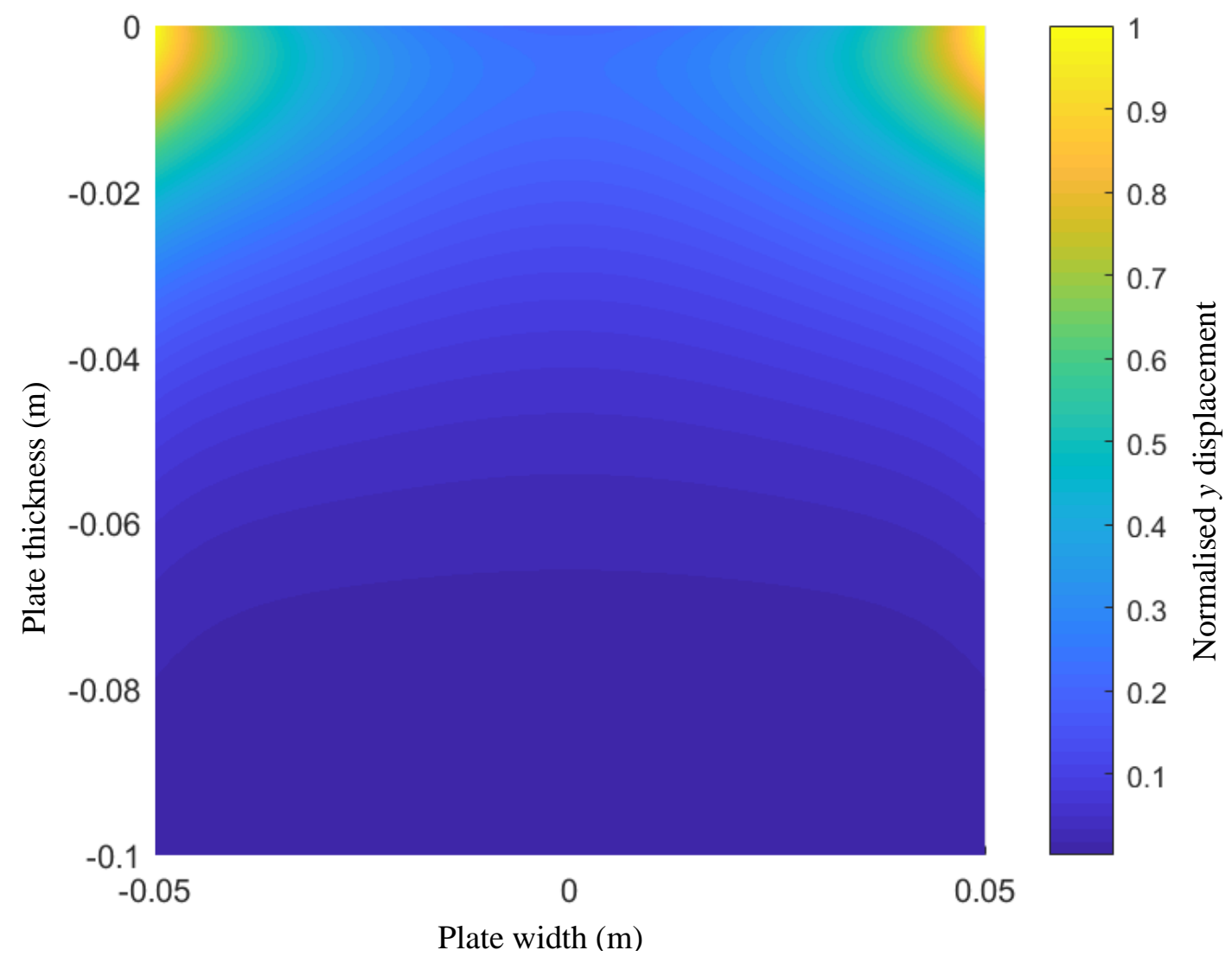




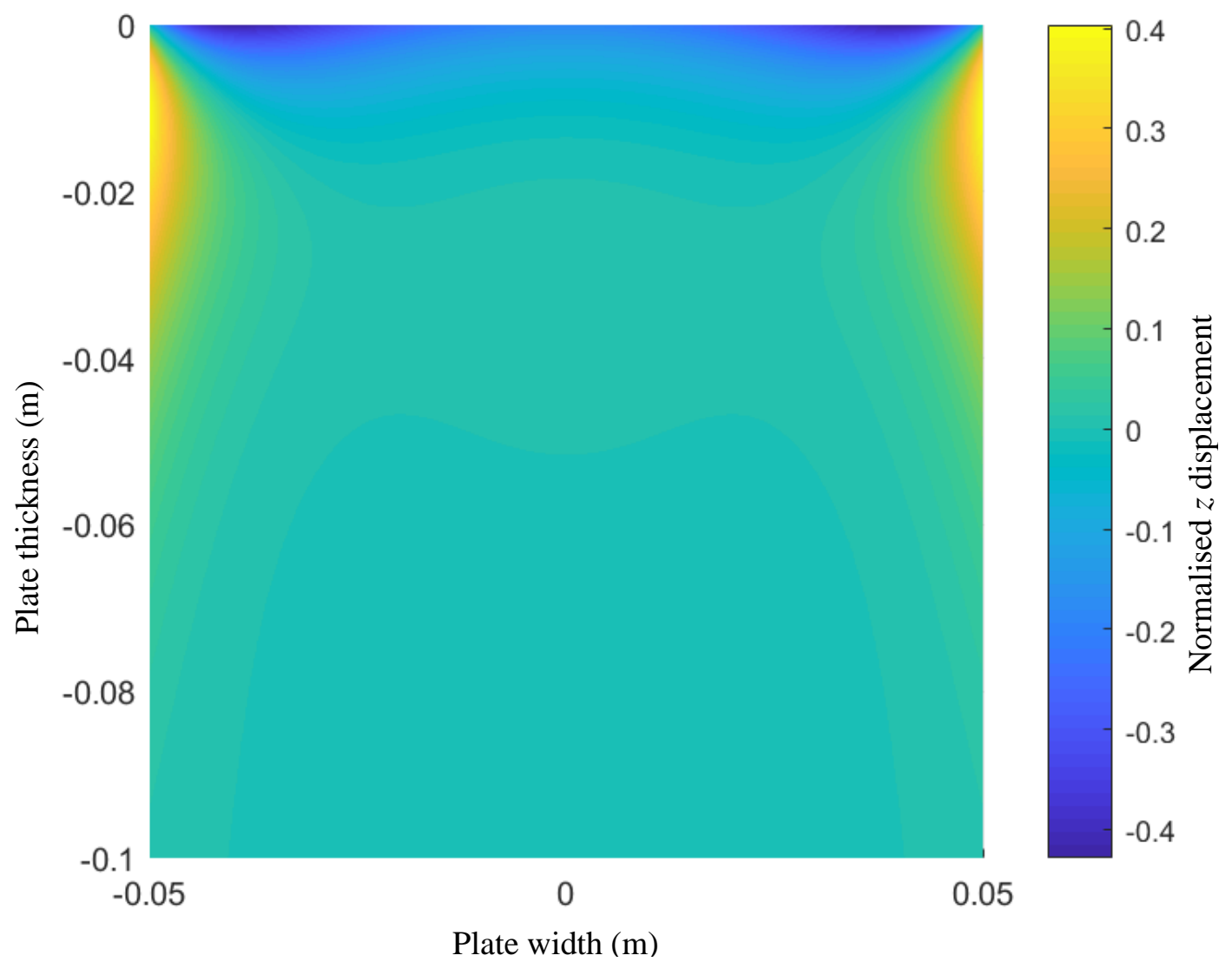

Fig. 7 Mode shape of the fundamental symmetric edge wave $\mathrm{E}_{\mathrm{S} 0}$ at $60 \mathrm{kHz}$. (a) $x$ displacement; (b) $y$ displacement; (c) $z$ displacement.

The mode shapes of the higher order modes are significantly different to mode shapes of the fundamental modes. For the fundamental modes $\mathrm{E}_{\mathrm{S} 0}$ and $\mathrm{E}_{\mathrm{A} 0}$, the displacements are either in phase or out of phase, similar to Lamb wave modes in a plate. However, higher order modes have phase variation across the cross-sectional area of the plate. The eigen vectors $u_{x}, u_{y}$ and $u_{z}$ (see Eq. 10) are complex for higher order modes, and they cannot be represented by a positive or negative real number. To display the mode shapes of these higher order modes, the magnitude of the displacements are taken and shown in Figs. 8 and 9. Furthermore, the magnitude of these modes shapes are symmetric with respect to $x=0$, and thus mode shapes are shown from $x=0$ to $x=0.05$, i.e., the positive side of the plate. 
The higher order flexural mode $\mathrm{E}_{\mathrm{A} 1}$ has leaky $x$ and $z$ displacement components. The $y$ displacement has energy focused near the top corners of the plate. Note that for leaky wave modes, modal amplitude decays in the wave propagation direction $z$, but grows in the transverse direction $y$ [41]. The higher order symmetric edge wave $\mathrm{E}_{\mathrm{S} 1}$ also has leaky $x$ and $z$ displacement components. The $y$ displacement has energy focused near the centre of the top surface of the plate.

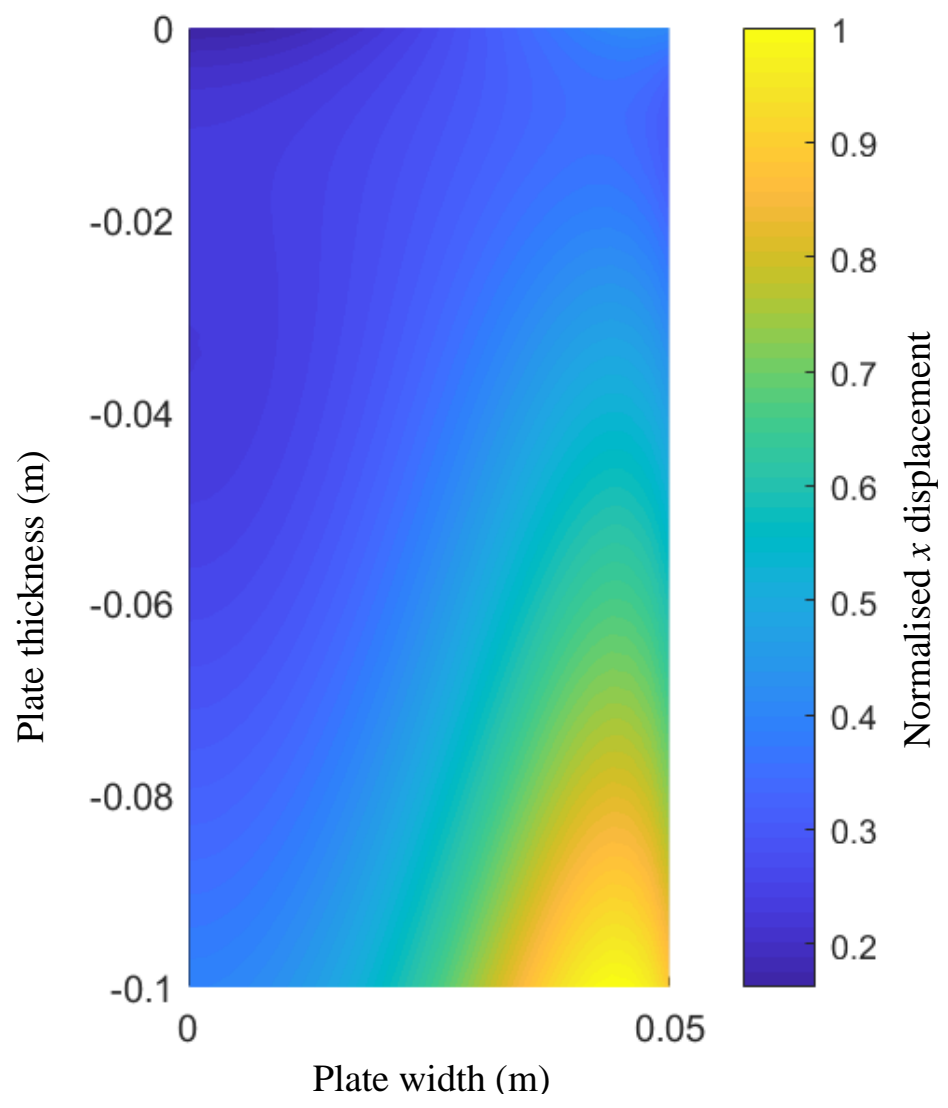




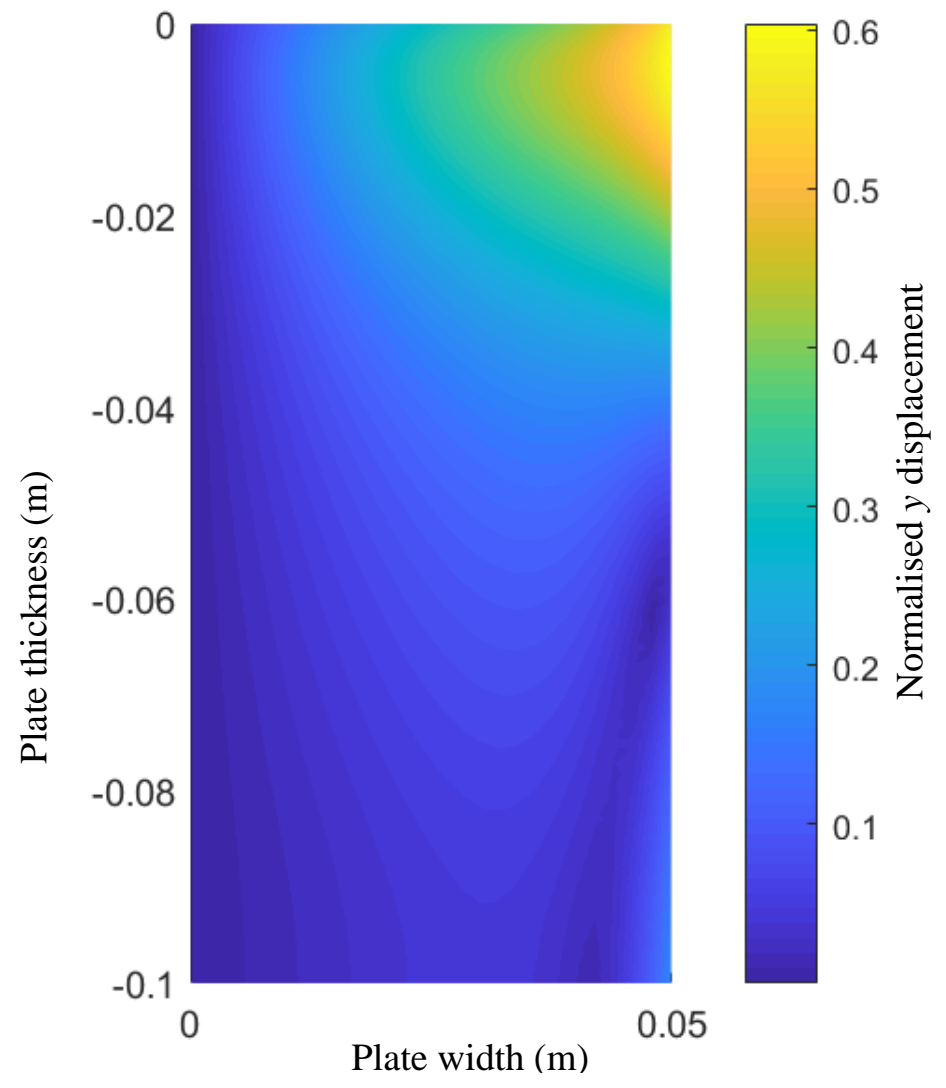




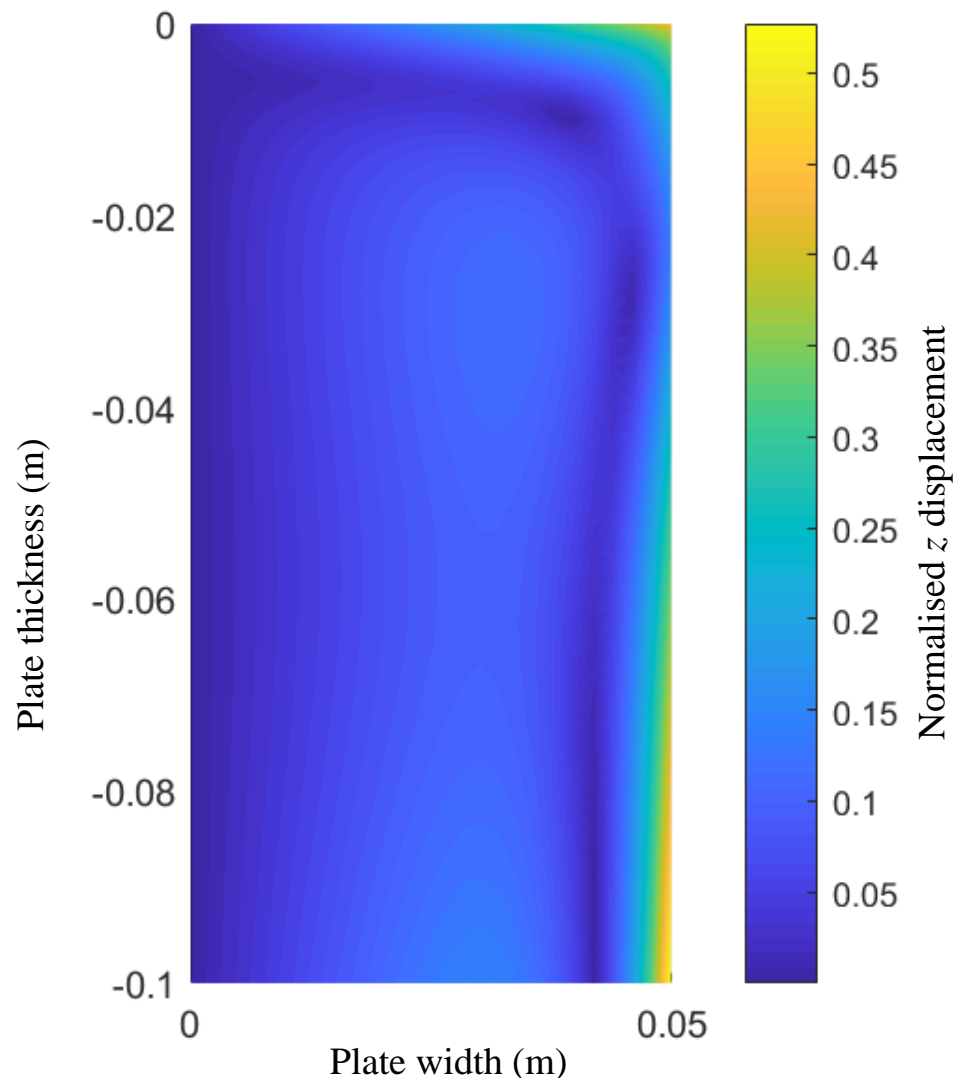

Fig. 8 Mode shape of the higher order flexural edge wave $\mathrm{E}_{\mathrm{A} 1}$ at $60 \mathrm{kHz}$. (a) $x$ displacement;

(b) $y$ displacement; (c) $z$ displacement. 


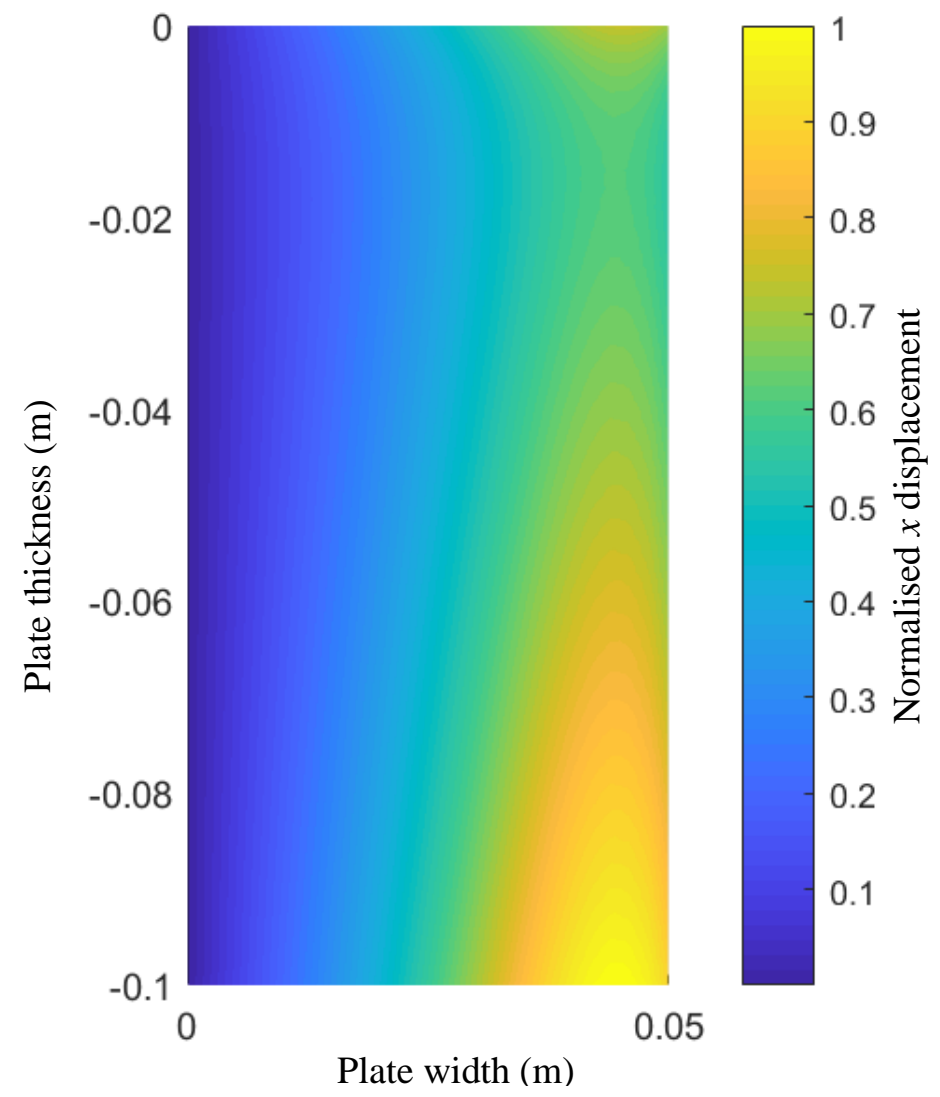




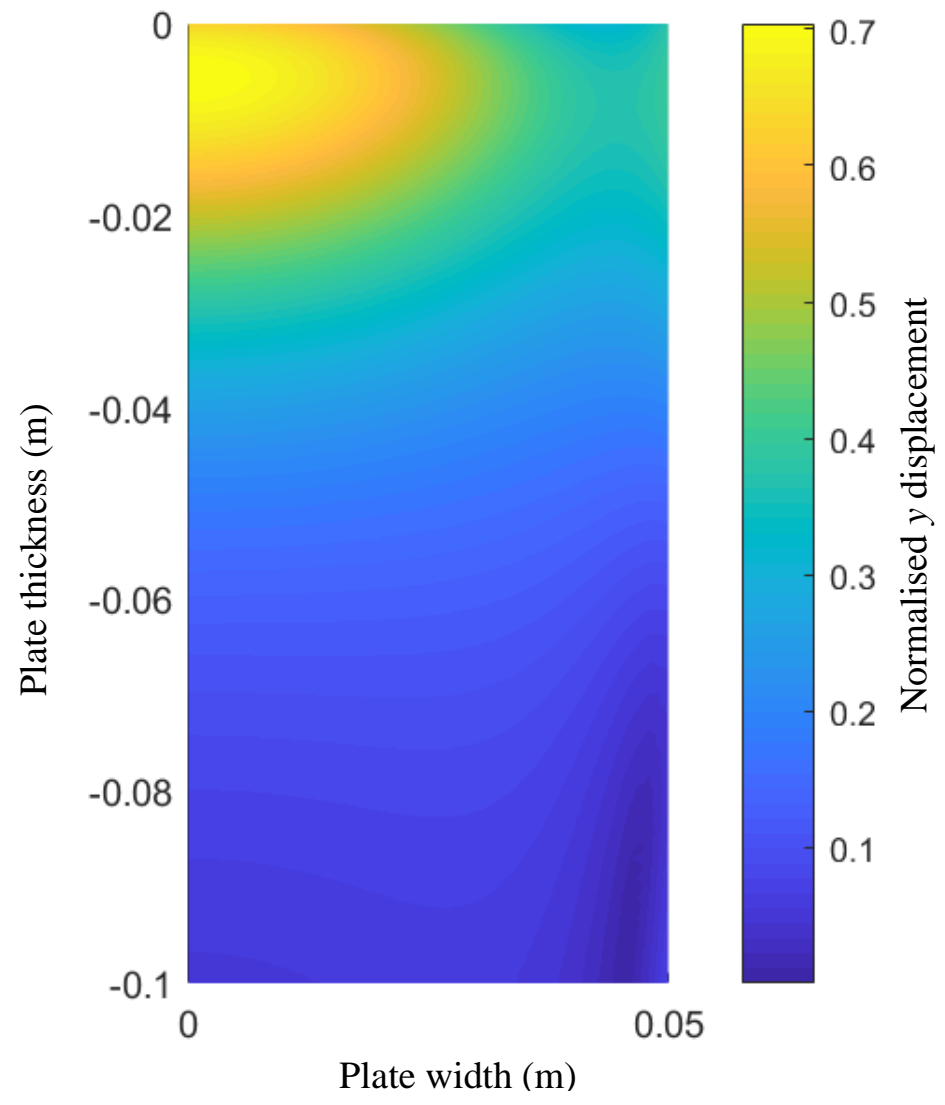




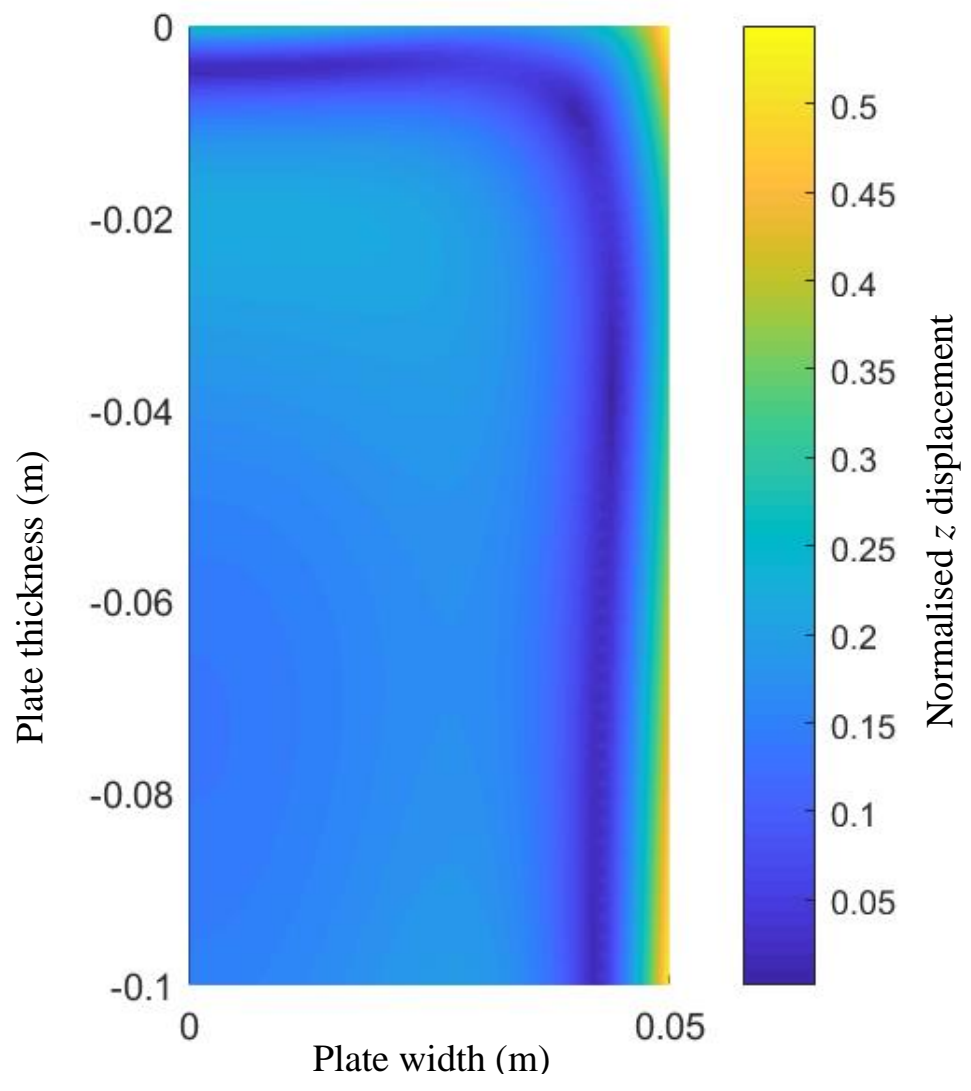

Fig. 9 Mode shape of the higher order symmetric edge wave $\mathrm{E}_{\mathrm{S} 1}$ at $60 \mathrm{kHz}$. (a) $x$ displacement; (b) $y$ displacement; (c) $z$ displacement.

\section{Conclusions}

In this paper, a two-dimensional semi-analytical finite element method has been proposed to study Rayleigh, Love and edge waves in three dimensional structures which may include multiple layers, as well as structures with infinite or finite widths. To avoid modelling an infinitely large region, a perfectly matched layer method has been used to close the computation domain. The model has been structured in a general way, so that both Lamb type and Shear-horizontal type surface waves can be calculated. Excellent agreement between the 
current numerical solutions and available Rayleigh, Love and fundamental edge wave solutions have been achieved.

On this basis, the full dispersion curves and mode shapes of a three dimensional finite width plate have been presented. It can be shown that fundamental edge wave modes are similar to conventional symmetric and anti-symmetrical Lamb wave modes in a plate. However, energy of these wave modes are totally focused near the top surface of the plate, i.e., trapped. Higher order edge waves have very different characteristics. Although the phase velocity of these wave modes are still less than the shear wave velocity of the plate material in the high frequency range, these wave modes are not trapped wave modes. It appears that the $x$ and $z$ components of the displacement behave as leaky wave modes. Only the vertical $y$ component of the displacement resembles that of a conventional edge wave mode, i.e., energy focused near the top of the plate.

Appendix

The matrices in Eq. (10) are given as:

$$
\begin{gathered}
Z_{1 x}=\left(\lambda_{p}+2 \mu_{p}\right) K_{x p}+\mu_{p} K_{y p}-\rho_{p} \omega^{2} K_{2 p}+\left(\lambda_{m}+2 \mu_{m}\right) K_{x m}+\mu_{m} K_{y m} \\
-\rho_{m} \omega^{2} K_{2 m} \\
Z_{1 y}=\lambda_{p} K_{x y p}+\mu_{p} K_{x y p}^{T}+\lambda_{m} K_{x y m}+\mu_{m} K_{x y m}^{T} \\
Z_{1 z}=-\lambda_{p} K_{3 p}^{T}+\mu_{p} K_{3 p}-\lambda_{m} K_{3 m}^{T}+\mu_{m} K_{3 m} \\
Z_{2 x}=\lambda_{p} K_{x y p}^{T}+\mu_{p} K_{x y p}+\lambda_{m} K_{x y m}^{T}+\mu_{m} K_{x y m} \\
Z_{2 y}=\left(\lambda_{p}+2 \mu_{p}\right) K_{y p}+\mu_{p} K_{x p}-\rho_{p} \omega^{2} K_{2 p}+\left(\lambda_{m}+2 \mu_{m}\right) K_{y m}+\mu_{m} K_{x m} \\
-\rho_{m} \omega^{2} K_{2 m}
\end{gathered}
$$




$$
\begin{gathered}
Z_{2 z}=-\lambda_{p} K_{4 p}^{T}+\mu_{p} K_{4 p}-\lambda_{m} K_{4 m}^{T}+\mu_{m} K_{4 m} \\
Z_{3 x}=-\lambda_{p} K_{3 p}+\mu_{p} K_{3 p}^{T}-\lambda_{m} K_{3 m}+\mu_{m} K_{3 m}^{T} \\
Z_{3 y}=-\lambda_{p} K_{4 p}+\mu_{p} K_{4 p}^{T}-\lambda_{m} K_{4 m}+\mu_{m} K_{4 m}^{T} \\
Z_{3 z}=-\mu_{p} K_{x p}-\mu_{p} K_{y p}+\rho_{p} \omega^{2} K_{2 p}-\mu_{m} K_{x m}-\mu_{m} K_{y m}+\rho_{m} \omega^{2} K_{2 m} \\
F_{x}=\mu_{p} K_{2 p}+\mu_{m} K_{2 m} \\
F_{z}=\left(\lambda_{p}+2 \mu_{p}\right) K_{2 p}+\left(\lambda_{m}+2 \mu_{m}\right) K_{2 m}
\end{gathered}
$$

In the SAFE layer,

$$
\begin{gathered}
K_{x p}=\int_{\Omega_{p}} \frac{\partial \mathbf{N}^{\mathrm{T}}}{\partial x} \frac{\partial \mathbf{N}}{\partial x} d \Omega_{p} \\
K_{x y p}=\int_{\Omega_{p}} \frac{\partial \mathbf{N}^{\mathrm{T}}}{\partial x} \frac{\partial \mathbf{N}}{\partial y} d \Omega_{p} \\
K_{y p}=\int_{\Omega_{p}} \frac{\partial \mathbf{N}^{\mathrm{T}}}{\partial y} \frac{\partial \mathbf{N}}{\partial y} d \Omega_{p} \\
K_{2 p}=\int_{\Omega_{p}} \mathbf{N}^{\mathrm{T}} \mathbf{N} d \Omega_{p} \\
K_{3 p}=\int_{\Omega_{p}} \mathbf{N}^{\mathrm{T}} \frac{\partial \mathbf{N}}{\partial x} d \Omega_{p} \\
K_{4 p}=\int_{\Omega_{p}} \mathbf{N}^{\mathrm{T}} \frac{\partial \mathbf{N}}{\partial y} d \Omega_{p}
\end{gathered}
$$

In the PML layer,

$$
K_{x m}=\int_{\Omega_{m}} \frac{\xi_{y}}{\xi_{x}} \frac{\partial \mathbf{N}^{\mathrm{T}}}{\partial x} \frac{\partial \mathbf{N}}{\partial x} d \Omega_{m}
$$




$$
\begin{aligned}
K_{x y m} & =\int_{\Omega_{m}} \frac{\partial \mathbf{N}^{\mathrm{T}}}{\partial x} \frac{\partial \mathbf{N}}{\partial y} d \Omega_{m} \\
K_{y m} & =\int_{\Omega_{m}} \frac{\xi_{x}}{\xi_{y}} \frac{\partial \mathbf{N}^{\mathrm{T}}}{\partial y} \frac{\partial \mathbf{N}}{\partial y} d \Omega_{m} \\
K_{2 m} & =\int_{\Omega_{m}} \xi_{x} \xi_{y} \mathbf{N}^{\mathrm{T}} \mathbf{N} d \Omega_{m} \\
K_{3 m} & =\int_{\Omega_{m}} \xi_{y} \mathbf{N}^{\mathrm{T}} \frac{\partial \mathbf{N}}{\partial x} d \Omega_{m} \\
K_{4 m} & =\int_{\Omega_{m}} \xi_{x} \mathbf{N}^{\mathrm{T}} \frac{\partial \mathbf{N}}{\partial y} d \Omega_{m}
\end{aligned}
$$

Here, $\mathbf{N}$ is the global trial (or shape) function written in the form of a row vector.

\section{References:}

1. Rayleigh, L., On waves propagated along the plane surface of an elastic solid. Proceedings of the London Mathematical Society, 1885. s1-17: p. 4-11.

2. Love, A.E.H., Some Problems of Geodynamics. 1911, Cambridge: University Press.

3. Y.K. Konenkov, A Rayleigh-type flexural wave, Soviet Physics-Acoustics, 6 (1960), pp. $122-123$

4. R.N. Thurston, J. McKenna, Flexural acoustic waves along the edge of a plate, IEEE Transactions on Sonics and Ultrasonics, SU-21 (4) (1974), pp. 296-297

5. B.K. Sinha, Some remarks on propagation characteristics of ridge guides for acoustic waves at low frequencies, Journal of the Acoustical Society of America, 56 (1974), pp. 16-18 
6. P.E. Lagasse, A.A. Oliner, Acoustic flexural mode on a ridge of semi-infinite height, Electronic Letters, 12 (1) (1976), pp. 11-13

7. A.N. Norris, Flexural edge waves, Journal of Sound and Vibrations, 171 (1994), pp. 571 573

8. I. D. Abrahamsand A. N. Norris, On the existence of flexural edgewaves on submerged elastic plates, Proc. R. Soc. London, Ser. A 456, 1559-1582 (2000).

9. A.N. Norris, V.V. Krylov, I.D. Abrahams, Flexural edge waves and comments on "A new bending wave solution for the classical plate equation" [J. Acoust. Soc. Am. 104, 2220-2222 (1998)], Journal of the Acoustical Society of America, 107 (3) (2000), pp. 1781-1784

10. D.D. Zakharov, Analysis of the acoustical edge flexural mode in a plate using refined asymptotics, Journal of the Acoustical Society of America, 116 (2) (2004), pp. 872-878

11. Y.B. Fu, D.W. Brookes, Edge waves in asymmetrically laminated plates, Journal of Mechanics and Physics of Solids, 54 (2006), pp. 1-21

12. P. Lu, H.B. Chen, H.P. Lee, C. Lu, Further studies on edge waves in anisotropic elastic plates, International Journal of Solids and Structures, 44 (2007), pp. 2192-2208

13. A. Milanese, P. Marzocca, M. Belubekyan, K. Ghazaryan, Effect of the stiffness and inertia of a rib reinforcement on localized bending waves in semi-infinite strips, International Journal of Solids and Structures, 46 (2009), pp. 2126-2135

14. G.T. Piliposian, M.V. Belubekyan, K.B. Ghazaryan, Localized bending waves in a transversely isotropic plate, Journal of Sound and Vibration, 329 (2010), pp. 3596-3605

15. J. Kaplunov, D. A. Prikazchikov, G. A. Rogerson, Edge bending wave on a thin elastic plate resting on a Winkler foundation, Proceedings of the Royal Society A, Mathematical, Physical and Engineering Sciences, London, UK (2016), Vol. 472, No. 2190.

16. J. Kaplunov, D.A. Prikazchikov, Asymptotic theory for Rayleigh and Rayleigh-type waves. Adv. Appl. Mech., 50 (2017) doi:10.1016/bs.aams.2017.01.001.

17. A.A. Krushynska, Flexural edge waves in semi-infinite elastic plates, J. Sound Vib., 330 (2011), pp. 1964-1976

18. J. Kaplunov, D.A. Prikazchikov, G.A. Rogerson, On three-dimensional edge waves in semi-infinite isotropic plates subject to mixed face boundary conditions, Journal of the Acoustical Society of America, 118 (5) (2005), pp. 2975-2983

19. V. Zernov, J. Kaplunov, Three-dimensional edge waves in plates, Proceedings of the Royal Society of London, Series A, A64 (2008), pp. 301-318 
20. V. Pagneux, Revisiting the edge resonance for lamb waves in a semi-infinite plate, J. Acoust. Soc. Am., 120 (2006), pp. 649-656

21. Postnova J., Craster R.V., Trapped modes in 3D topographically varying plates. IMA J. Appl. Math. 73 (2008), 950-963

22. Z. Fan and M. J. S. Lowe, Elastic waves guided by a welded joint in a plate, Proc. R. Soc. London, Ser. A 465, 2053-2068 (2009)

23. Z. Fan, M. Castaings, M.J. Lowe, C. Biateau, P. Fromme, Feature-guided waves for monitoring adhesive shear modulus in bonded stiffeners, NDT\&E Int., 54 (2013), pp. 96-102

24. A. Ramdhas, R.K. Pattanayak, K. Balasubramaniam, P. Rajagopal, Symmetric lowfrequency feature-guided ultrasonic waves in thin plates with transverse bends, Ultrasonics, 56 (2015), pp. 232-242

25. X. Yu, P. Manogharan, Z. Fan, P. Rajagopal, Shear horizontal feature guided ultrasonic waves in plate structures with 90 transverse bends, Ultrasonics, 65 (2016), pp. 370-379

26. J. Barshinger, J.L. Rose, M.J. Avioli Jr, Guided wave resonance tuning for pipe inspection, J. Press. Vess. T. 124 (2002) 303-310.

27. T. Hayashi, W-J. Song, J.L. Rose, Guided wave dispersion curves for a bar with an arbitrary cross-section, a rod and rail example, Ultrasonics 41 (2003) 175-183.

28. A. Marzani, E. Viola, I. Bartoli, F. Lanza di Scalea, P. Rizzo, A semi-analytical finite element formulation for modelling stress wave propagation in axisymmetric damped waveguides, J. Sound Vib. 318 (2008) 488-505.

29. F. Treyssède, L Laguerre, Numerical and analytical calculation of modal excitability for elastic wave generation in lossy waveguides, J. Acoust. Soc. Am. 133 (2013) 3827-3837.

30. Duan, W., Niu, X., Gan, TH., Kanfoud, J. and Chen, HP, A numerical study on the excitation of guided waves in rectangular plates using multiple point sources. Metals (2017), 7 (12) 552

31. K. L. Nguyen, F. Treyssède, C. Hazard, Numerical modeling of three-dimensional open elastic waveguides combining semi-analytical finite element and perfectly matched layer methods, Journal of Sound and Vibration, 344 (2015) 158 - 178

32. W. Duan, R. Kirby, P. Mudge, T.H. Gan, A one dimensional numerical approach for computing the eigenmodes of elastic waves in buried pipelines, J. Sound Vib., 384 (2016), pp. $177-193$ 
33. P. Zuo, Z. Fan, SAFE-PML approach for modal study of waveguides with arbitrary cross sections immersed in inviscid fluid, J Sound Vib, 406 (2017), pp. 181-196

34. Kalkowski M.K., Muggleton M.J., Rustighi E. Axisymmetric semi-analytical finite elements for modelling waves in buried/submerged fluid-filled waveguides. Computers and Structures 2018; 196: 327-340.

35. Foti S. Multi-station methods for geotechnical characterisation using surface waves. $\mathrm{PhD}$ Thesis., Politecnico di Torino; 2000.

36. Hisada, Y. An Efficient Method for Computing Green's Functions for a Layered HalfSpace with Sources and Receivers at Close Depths (Part 2), Bulletin of the Seismological Society of America, 85(4), 1995 1080-1093

37. W. Duan, R. Kirby, A numerical model for the scattering of elastic waves from a nonaxisymmetric defect in a pipe, Finite Elem. Anal. Des., 100 (2015), pp. 28-40

38. Eslick R, Tsoflias G, Steeples DW (2008) Field investigation of Love waves in nearsurface seismology. Geophysics 73(3):G1-G6

39. Aki, K., P. G. Richards, Qualitative Seismology: theory and methods, University Science Books, 2002

40. M. Romeo, Rayleigh waves on a viscoelastic solid half-space, J. Acoust. Soc. Am., 110 (2001), pp. 59-67

41. T. Vogt, M. Lowe, P. Cawley, The scattering of guided waves in partly embedded cylindrical structures, J. Acoust. Soc. Am., 113 (3) (2003), pp. 1258-1272 